\title{
Enteroscopy in small-bowel Crohn's disease (CD): hypes and hopes
}

Authors

Institution
Krisztina B. Gecse, Peter L. Lakatos

1st Department of Medicine, Semmelweis University, Budapest, Hungary submitted

15. September 2014 accepted after revision 26. September 2014

\section{Bibliography}

DOI http://dx.doi.org/ 10.1055/s-0034-1390881 Endoscopy International Open 2014; 02: E199-E200

(c) Georg Thieme Verlag KG Stuttgart . New York E-ISSN 2196-9736

\section{Corresponding author}

\section{Peter L. Lakatos, MD, PhD}

1st Department of Medicine Semmelweis University Korányi Sándor street 2/a 1083 Budapest Hungary

Fax: +36 13130250

lakatos.peter_laszlo@med. semmelweis-univ.hu
Crohn's disease (CD) is a chronic inflammation of the intestines that follows a relapsing or remitting course. Endoscopic imaging and radiological imaging are essential for diagnosis and to assess disease severity, response to treatment, and complications. CD most commonly affects the terminal ileum, which is accessible with conventional ileocolonoscopy. Nevertheless, jejunal lesions are present in more than half of Crohn's patients and are associated with an increased risk of clinical relapse [1]. The presence of proximal small-bowel lesions has an impact on prognosis and therapeutic management, with early introduction of immunomodulators and biologicals [2]. Several modalities are available for small-bowel imaging. However, a standardized algorithm is lacking and the choice of modality is largely dependent on local availability and expertise.

A prospective, blinded study compared multiple small-bowel imaging modalities after ileocolonoscopy in 93 patients with suspected or established CD [3]. The sensitivity and specificity for terminal ileal CD were 100 and $91 \%$ for smallbowel capsule endoscopy (SBCE), 81 and $86 \%$ for magnetic resonance (MR) enterography, and 76 and $85 \%$ for computed tomography (CT) enterography, respectively. Additionally, SBCE significantly enhanced the detection of proximal small-bowel lesions [3]. According to a recent meta-analysis, no significant difference was found between the sensitivity and specificity of CT enterography and MR enterography to detect active small-bowel CD, and accuracy was largely similar for ultrasound (US) in an earlier systematic review [4]. SBCE is considered the most sensitive test to exclude early small-intestinal lesions, with a negative predictive value of $96 \%$ [5]. The risk of capsule retention is relatively low in patients with suspected CD in the absence of obstructive symptoms. On the contrary in patients with established $C D$, retention risk significantly increases. Small-bowel US is increasingly used for preliminary assessment and during the follow-up of patients with potential inflammatory bowel disease (IBD). The advantages of abdominal US include easy access, low cost, non-invasiveness, and lack of ionizing radiation, although the accuracy is highly dependent on the available expertise. The currently ongoing MREnterography or ulTRasound in Crohn disease (METRIC) study prospectively compares the diagnostic efficacy, therapeutic impact, and cost-effectiveness of MR enterography and small-bowel US in relapsing $\mathrm{CD}$.

In the present issue of the journal, Navaneethan et al. [6] aim to evaluate the role of balloon-assisted enteroscopy (BAE) in 65 patients with suspected and established small-bowel CD after a complete evaluation including CT or MR enterography. Interestingly, in the group of patients with suspected $C D(n=22)$, enteroscopy increased the diagnostic specificity of SBCE and confirmed CD in 3 patients whose lesions seemed nonspecific upon SBCE. Additionally, in the group of patients with established $C D(n=43)$, active inflammation was identified in 18 patients, which led to escalation of medical therapy. Endoscopic balloon dilation (EBD) of fibrotic stenosis was successfully carried out in 5 patients. Of note, the agreement between CT or MR enterography and BAE in suspected $C D$ was only modest (36.4\%), with normal BAE findings in the majority of the patients with increased wall thickness. In contrast, in established CD a large proportion of the lesions were detected already with conventional imaging (overall agreement with CT or MR enterography was $75.6 \%$ ). Nonetheless, although this is one of the largest series evaluating the additive value of BAE in the management of $C D$ patients, results should still be considered as preliminary; and BAE should be reserved for patients with unexplained symptoms and laboratory alterations suggestive of small-bowel CD or complications of 
CD after a complete evaluation by conventional imaging techniques.

BAE is an invasive imaging method imposing significant burdens on patients, endoscopists, and health care providers. Its current use is therefore restricted to when enteroscopy is expected to alter management strategy (i.e., to confirm diagnosis), or to invasive procedures (e.g., therapeutic balloon dilation). In patients with suspected $C D$ with clinical expression limited to the small bowel, double-balloon enteroscopy (DBE) may be a helpful device. Nevertheless, the combination of clinical findings and additional imaging modalities is required to confirm the diagnosis. Earlier studies have also demonstrated that BAE has an additive value in the management of $C D$, leading to a change in the therapeutic strategy in up to 30 to $60 \%$ of patients with suspected small-bowel lesions [7].

Finally, the technical success for EBD has been reported to vary from 86 to $93 \%$, with the clinical success (resolution of obstructive symptoms) varying from 64 to $70 \%[8,9]$. Upon long-term follow-up, stricture often recurs. Re-dilatations may be required in up to $20 \%$ of patients at 1 year and up to $50 \%$ of patients by 5 years. The cumulative proportion of patients needing surgery at 1 and 5 years is approximately 15 and $40 \%$, respectively. In comparison, recurrence rate after strictureplasty is approximately $45 \%$ at 5 -year follow-up. Major complications, such as bowel perforation and significant bleeding, occur in about $2 \%$ of patients, which is again comparable to the $5 \%$ of the surgery-related complication rate. Future options may include endoscopic treatment of strictures with biodegradable stent implantation. Although recently published case reports seem promising, further data are needed to evaluate their feasibility in CD strictures.

In conclusion, proximal small-bowel involvement has a major impact on disease prognosis and therapeutic strategies. Therefore, the recognition of small-bowel lesions is essential. In patients with suspected CD, SBCE has the highest diagnostic yield for the detection of small-intestinal lesions. Patients' acceptance is also high. Thus, it may be the initial imaging method, in the absence of obstructive symptoms [10]. Should imaging remain inconclusive, enteroscopy offers the additional benefits of biopsy sampling and histological examination to confirm diagnosis. In patients with established $\mathrm{CD}$, MR or CT enterography is preferable, due to their ability to characterize stenosis as well as extraluminal complications [10]. In the presence of short $(<4 \mathrm{~cm})$, non-angulated fibrotic strictures, enteroscopy also has a potential for therapeutic intervention. The success and safety of EBD are comparable to that of surgery; and recurrence rates are similar.

\section{Competing interests: None}

\section{References}

1 Flamant $M$, Trang $C$, Maillard $O$ et al. The prevalence and outcome of jejunal lesions visualized by small bowel capsule endoscopy in Crohn's disease. Inflamm Bowel Dis 2013; 19: 1390-1396

2 Cotter J, Dias de Castro F, Moreira MJ et al. Tailoring Crohn's disease treatment: the impact of small bowel capsule endoscopy. J Crohns Colitis DOI 10.1016/j.crohns.2014.02.018 [Epub ahead of print]

3 Jensen MD, Nathan T, Rafaelsen SR et al. Diagnostic accuracy of capsule endoscopy for small bowel Crohn's disease is superior to that of MR enterography or CT enterography. Clin Gastroenterol Hepatol 2011; 9: $124-129$

4 Qiu Y, Mao R, Chen BL et al. Systematic review with meta-analysis: magnetic resonance enterography vs. computed tomography enterography for evaluating disease activity in small bowel Crohn's disease. Aliment Pharmacol Ther 2014; 40: 134-146

5 Leighton JA. The role of endoscopic imaging of the small bowel in clinical practice. Am J Gastroenterol 2011; 106: 27-36

6 Navaneethan U, Vargo IJ, Menon KVN et al. Impact of balloon-assisted enteroscopy on the diagnosis and management of suspected and established small bowel Crohn's disease. EIO 2014; 2: 201 -206

7 Mensink PB, Aktas H, Zelinkova Z et al. Impact of double-balloon enteroscopy findings on the management of Crohn's disease. Scand J Gastroenterol 2010; 45: 483-489

8 Hassan C, Zullo A, De Francesco V et al. Systematic review: endoscopic dilatation in Crohn's disease. Aliment Pharmacol Ther 2007; 26: $1457-1464$

9 Hirai F, Beppu T, Takatsu $N$ et al. Long-term outcome of endoscopic balloon dilation for small bowel strictures in patients with Crohn's disease. Dig Endosc 2014; 26: 545-551

10 Annese V, Daperno M, Rutter MD et al. European evidence based consensus for endoscopy in inflammatory bowel disease. J Crohns Colitis 2013; 7: $982-1018$ 